\title{
Comparison between the outcome of single versus multiple uterine fibroids after uterine artery embolization
}

\author{
Ahmed M. Elsharkawy ${ }^{1,2^{*}}$ (D, Haytham H. Elsaeed ${ }^{1}$, Ahmed H. Kamel ${ }^{3}$, Robert Morgan ${ }^{2}$ and Mohamed H. Elshafey ${ }^{1}$
}

\begin{abstract}
Background: Uterine fibroids are the most common benign tumor of the reproductive tract in women. Uterine artery embolization has been approved as an alternative treatment option for uterine fibroids to reduce uterine and fibroid volumes as well as to relieve the patient symptoms. The current study included 80 patients with symptomatic uterine fibroids. All patients underwent pre-embolization MRI, uterine artery embolization, and followup post-embolization MRI at 6 months post procedure for a reduction in uterine and dominant fibroid volumes. The aim of the study was to assess the role of transcatheter uterine artery embolization in reducing the uterine fibroid volume.

Results: There was a significant difference in the uterine volume reduction between a group with single fibroids and a group with multiple uterine fibroids The mean uterine volume reduction in the first group was $46.5 \%$ compared to $37.4 \%$ reduction in the second group. While there was no significant difference in the dominant fibroid volume reduction between both groups ( $48.8 \%$ and $44.9 \%$ respectively).

Conclusions: UAE has a high efficacy in the reduction of both the uterine volume and the dominant fibroid volume, which results in improvement in patient's quality of life.
\end{abstract}

Keywords: Fibroids, Uterine artery embolization, Gynecologic interventions

\section{Background}

Uterine fibroids (UF) are the commonest benign tumor in women of reproductive age [1]. Fibroids may vary in size from a few millimeters to large sizes (over $10 \mathrm{~cm}$ ) and can be classified into single or multiple uterine fibroids [2].

Uterine artery embolization (UAE) is an interventional radiological (IR) technique to occlude the arterial supply to the uterus for various reasons [3].

The technique was first reported as an effective technique for the treatment of uterine fibroids in 1995, when

\footnotetext{
* Correspondence: Ahmed.Elsharkawy84@gmail.com

${ }^{1}$ Radio-diagnosis \& Medical Imaging Department, Faculty of Medicine, Tanta University, Tanta, Egypt

2Diagnostic and Interventional Radiology Department, St George's University Hospital and NHS Foundation Trust, London, UK

Full list of author information is available at the end of the article
}

it was noted that women with symptomatic fibroids who underwent UAE as a pre-hysterectomy treatment had significant clinical improvement to the extent that hysterectomy was no longer required [4].

UAE has proved to be an effective treatment for symptomatic fibroids and it is now considered as an alternative to myomectomy and hysterectomy $[5,6]$. Standard imaging protocols for UAE are the pre-procedural assessment by magnetic resonance imaging (MRI) followed by a post-procedural MRI 3-6 months later [7, 8].

Embolization procedures are performed with 4F RIM catheter, microcatheters, and a variety of embolic agents. One of the decisions that an operator must make before performing a uterine artery embolization procedure is which embolic agent to use. Originally, irregularly shaped polyvinyl alcohol (PVA) particles were used, 
although more recently other spherical-shaped embolic agents have been developed $[9,10]$.

Infarction of a fibroid is indicated by the absence of contrast enhancement in follow-up T1-weighted MR images in comparison to pre-embolization MRI images. The degree of infarction is expressed by using a scale from 0 to $100 \%$ infarction, where $100 \%$ is complete infarction and $0 \%$ is non-infarction [11].

Persistent symptoms after UFE can be due to incomplete fibroid infarction, and the subsequent regrowth of an incompletely treated fibroid contributes to earlier symptom recurrence [12].

\section{Methods}

Eighty female patients were referred for UAE for symptomatic fibroids. UAE for fibroids was decided after discussion of treatment options in a fibroid multidisciplinary team meeting. Exclusion criteria were a positive pregnancy test, uterine or adnexal infection, suspected gynecologic malignancy, patients with renal impairment, and hypersensitivity to contrast media.

Patients were examined in both gynecology and IR clinics with a full detailed history, including the menstrual history, duration and amount of bleeding, and full laboratory investigations. Informed consent was obtained from all patients and patient information leaflets were given to them during the clinic visit.

\section{Pre and post procedure pelvic MRI}

Pelvic MRI was performed using a GE Signa Excite (GE Medical Systems, USA) 1.5 Tesla MRI scanner. Pre- and post-interventional MRIs were performed according to standard identical protocols.

The MRI sequences used in the study were sagittal T2 fast spin echo (FSE), axial T2 FSE, axial T1 FSE, and post contrast axial T1 fat-saturated imaging. Assessment criteria were the site and number of fibroids, the calculation of pre and post embolization uterine and dominant fibroid volumes, and the estimation of the percentage of dominant fibroid infarction on the post embolization MRI scans.

Clinical symptomatic improvement was assessed with by calculation of symptom severity score and a healthrelated quality of life questionnaire (HR-QOL) before and 6 months after UAE.

\section{Technique of uterine artery embolization}

The procedure was done under fluoroscopic guidance using a Siemens Artis-Q Angiography machine with a ceiling mounted system. The machine is capable of serial radiography and digital subtraction. Non-ionic contrast media (Omnipaque 300) was used in all patients (Figs. 1 and 2).
The embolization procedure was performed by certified interventional radiologists in our institution (R.M. with 30 years experience in interventional radiology, A.K. 15 years experience, and A.E. 10 years experience). Each operation was performed by two operators to decrease operative time and radiation exposure.

The patient is positioned in the supine position, and both groins are prepped with antiseptic solution. Bilateral common femoral arteries access is achieved using the Seldinger technique after infiltration of local anesthesthetic agent (10 $\mathrm{ml}$ lidocaine) around the femoral artery.

Then 4F Rim catheters (Rosch Inferior Mesenteric Torcon Beacon Tip catheter, Cook Medical, USA) are advanced over the aortic bifurcation over 0.035 hydrophilic guidewires (Radiofocus ${ }^{\oplus}$ Terumo) to reach the contra-lateral internal iliac artery. Catheterization of the uterine artery is then performed, and the catheter tip is placed in the transverse portion of the uterine artery. Arteriography is performed to confirm a satisfactory position.

In some patients, it was difficult to access the correct position with the Rim catheter. In these cases, a $2.7 \mathrm{~F}$ microcatheter (Progreat 2.7F microcatheter, Terumo Medical Corporation, Europe) was used to go further to access the appropriate position. Pulsed fluoroscopy (2 pulses per second) is used to opacify the entire fibroid uterus and to decrease patient radiation dose.

Embolization is started using 355 to $500 \mu \mathrm{m}$ nonspherical polyvinyl alcohol particles (Contour PVA, Boston Scientific, USA), which are then upsized to 500 to $710 \mu \mathrm{m}$ and $710-1000 \mu \mathrm{m}$.

The end point for embolization is stasis of contrast within the transverse segments of the uterine arteries for approximately 10 cardiac pulsations.

The operators usually wait for a period of $5 \mathrm{~min}$ to allow any remaining embolic material clumping to redistribute, and they then reconfirm adequate stasis by performing repeat angiography.

Following UAE, patient-controlled analgesia with the administration of intravenous analgesia and antiinflammatory drugs may be used to assist in pain control in the early post procedure period.

After UAE, patients are observed overnight in the hospital to ensure adequate access to pain and anti-nausea medications and to enable observation of the femoral access site.

\section{Merits and limitation of technique}

The merits of the uterine fibroid embolization are as follows: avoidance of hysterectomy, minimally invasive technique, shorter hospital stay, and shorter recovery time. However, this technique cannot be used in patients with uterine or adnexal infections, renal impairment, 

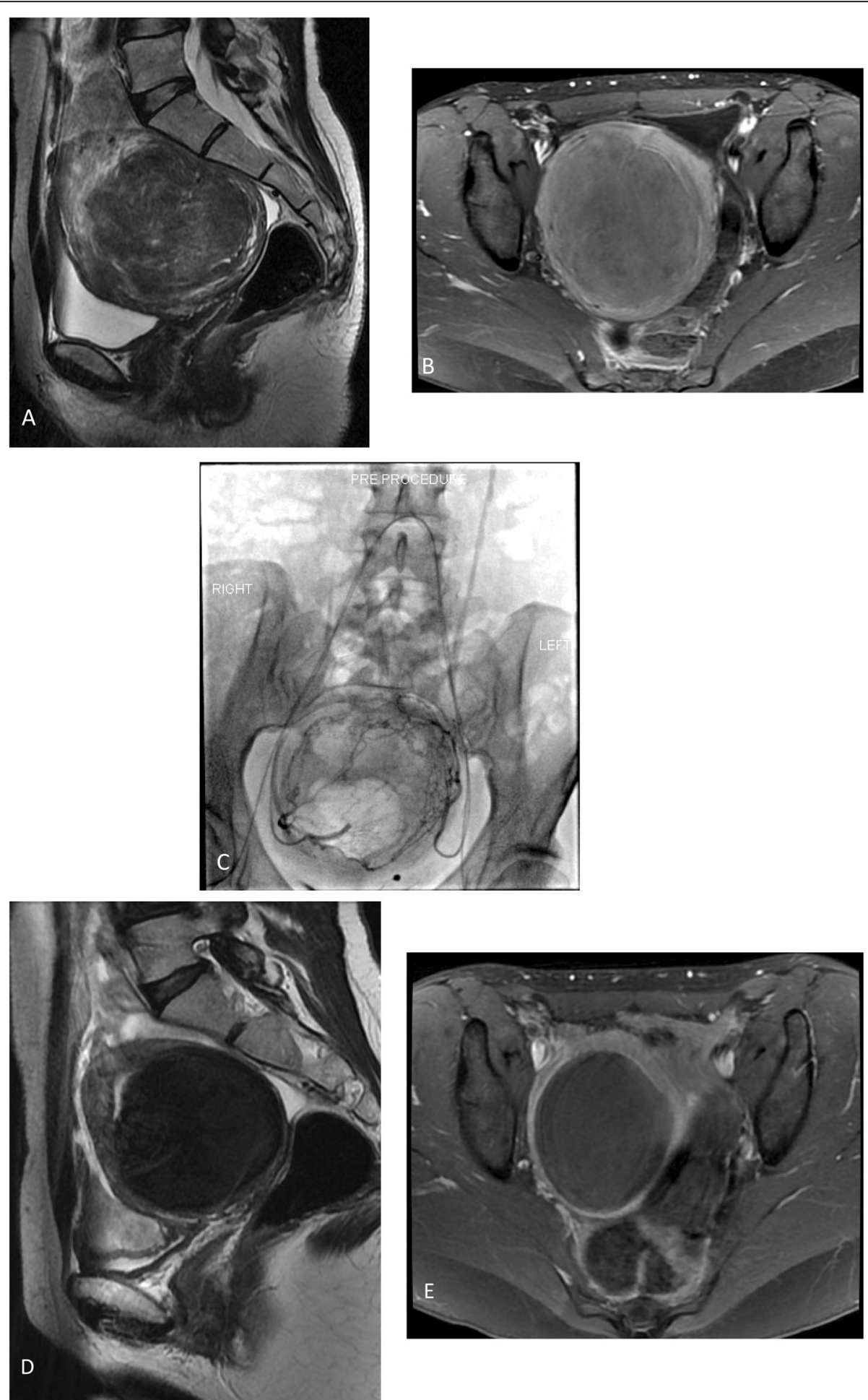

Fig. 1 Forty year-old-female patient. a Sagittal T2-weighted MR image shows a single large intramural fibroid. b A baseline MRI scan shows intense enhancement of the fibroid. c Uterine arteriograms before embolization: Fluoroscopic image obtained in the arterial phase shows bilateral hypertrophied uterine arteries with a large fibroid uterus due to uterine fibroids. $\mathbf{d}$ Sagittal T2-weighted MRI 6 months following uterine artery embolization demonstrates a reduction in the uterine and fibroid volume. e Post embolization T1-weighted MR image shows 100\% infarction with decreased fibroid volume 

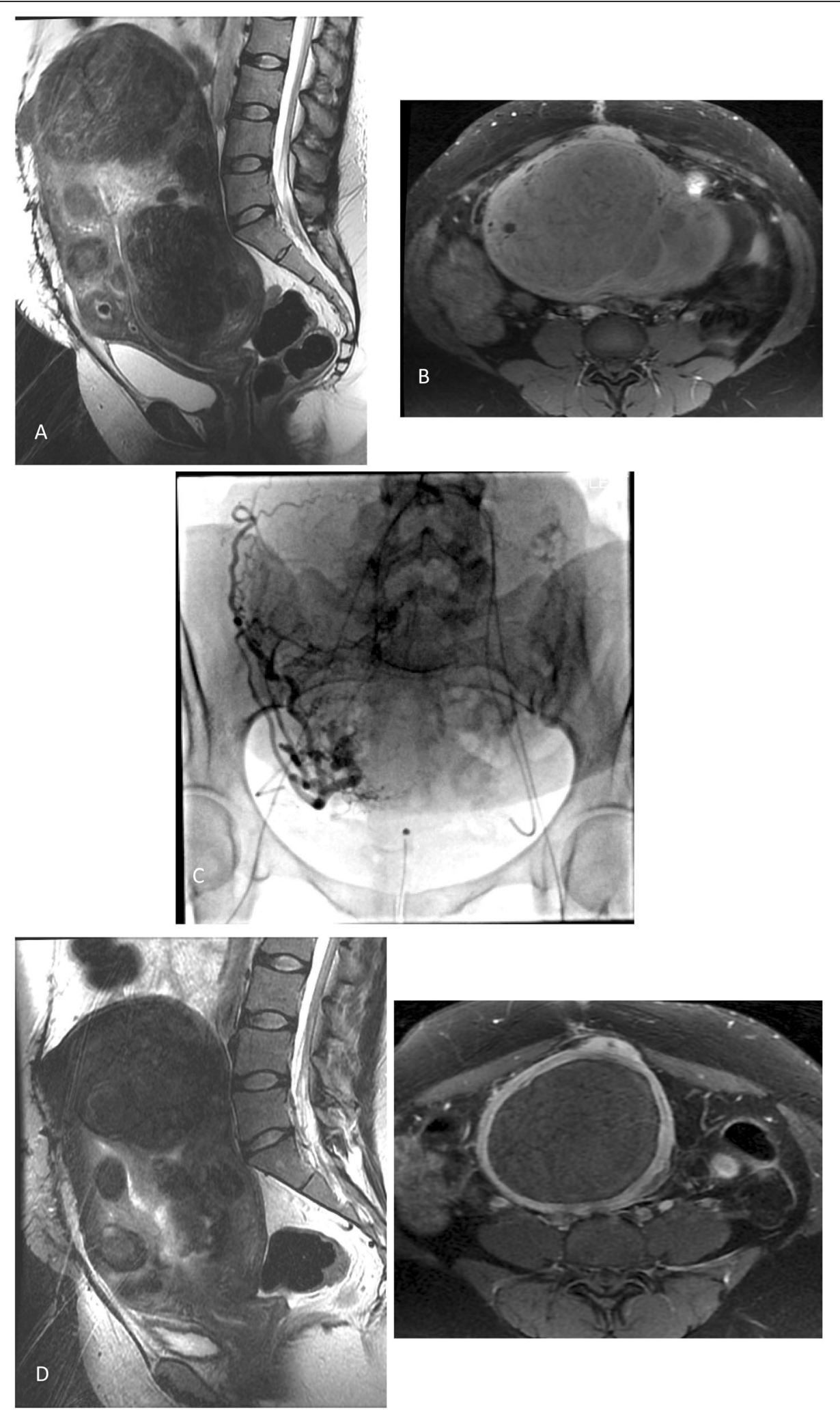

Fig. 2 (See legend on next page.) 
(See figure on previous page.)

Fig. 2 Thirty-five year-old-female patient. a Sagittal T2-weighted MR image demonstrates multiple variably sized uterine fibroids. b Baseline T1weighted fat-suppressed image shows an intense enhancement of the uterine fibroids. c Uterine angiograms before embolization: fluoroscopic image obtained in the arterial phase shows bilateral enlarged uterine arteries with an enlarged uterus due to multiple fibroids. $\mathbf{d}$ Post embolization T1-weighted fat-suppressed image shows 100\% infarction of the dominant fibroid and decreased uterine and fibroid volumes. e MRI scan 6 months post UAE shows a reduction in the uterine and fibroid volumes

and patients with known history of hypersensitivity to contrast media.

\section{Statistical analysis}

The collected data were coded, tabulated, and statistically analyzed using the SPSS program (Statistical Package for Social Sciences) software version 25.

Descriptive statistics were done for parametric quantitative data by mean, minimum, and maximum of the range, while they were done for categorical data by number and percentage.

Analyses were done for parametric quantitative data using paired samples $T$ test.

The level of significance was taken at a $P$ value of $<0.05$.

\section{Results}

Eighty patients with uterine leiomyomata were referred to the IR department between February 2018 and October 2019. They were recruited into the study after fulfillment of the inclusion criteria; their ages ranged from 34 to 54 years old; the patients were classified into 2 groups: 21 patients in the first group (group 1) had a solitary large fibroid, and 59 patients in the second group (group 2) had multiple fibroids. Patients complained of uterine bleeding and/or pelvic pressure symptoms in the form of pelvic heaviness and urinary disturbance (as in Table 1).

There was a significant difference in the percentage of uterine volume reduction between the two groups. In group 1, the pre-embolization uterine volume was 179$3628 \mathrm{~cm}^{3}$ with a mean volume $=608.1 \mathrm{~cm}^{3}$ and total volume reduction was $46.5 \%$. The pre-embolization total uterine volume in group 2 was $84.3-2179 \mathrm{~cm}^{3}$ with a mean volume $=872.9 \mathrm{~cm}^{3}$ and total volume reduction was $37.4 \%$ (significant difference, $P=0.01$ ) (as shown in Table 2).

Table 1 Number, age, and complaints of the two studied groups

\begin{tabular}{lll}
\hline & Treatment group & \\
\cline { 2 - 3 } & Single fibroid & Multiple fibroids \\
\hline Number of patients & 21 & 59 \\
Age (mean) & 42.7 & 43.3 \\
Menorrhagia & 16 & 39 \\
Pelvic pressure & 10 & 22 \\
\hline
\end{tabular}

However, there was no significant difference of the volume reduction of the dominant fibroid between the 2 groups. The pre-embolization dominant fibroid volume in group 1 group ranged from $52-290 \mathrm{~cm}^{3}$ (mean $=270$ $\mathrm{cm}^{3}$ ), and the volume reduction was $48.8 \%$. In group 2, the pre-embolization volume ranged from $23-170 \mathrm{~cm}^{3}$ $\left(\right.$ mean $\left.=247 \mathrm{~cm}^{3}\right)$ and the volume reduction was $44.9 \%$ (no significant difference, $P=0.57$ ) (as shown in Table 3).

- The number of PVA vials that were used in group 1 ranged from 2-10 vials with a mean of 4.7 vials, while in group 2, the number of PVA vials that were used ranged from 2-12 vials with a mean of 5 vials.

- As regarding procedural complications, expulsion of the infarcted fibroid occurred in 2 patients who presented with severe colicky pain. No major complication or puncture site hematoma was encountered.

- The mean screening time was $15.68 \mathrm{~min}$; the mean radiation dose was $191 \mathrm{mGy}$; and there was no significant difference in the dose area product (DAP) of $2520 \mu \mathrm{Gym}^{2}$, fluoroscopy time, or radiation dose between the two groups.

- In group 2, failure to cannulate the left uterine artery by the RIM catheter was experienced in 5 patients and the right uterine artery in one patient with the result that microcatheters were used in these patients. In group 1, there was failure to catheterize the left uterine artery with the RIM catheter in one patient, and a microcatheter was used successfully.

- In group 1 , the mean symptom severity score was 65.8 before UAE and was 30.4 after UAE. The mean HR-QOL before UAE was 33.3, was 78.2 after UAE, which was not statistically significant different $(P$ value $=0.67$ ) from group 2 . In group 2 , the mean pre-UAE symptom severity score was 70.1 and was

Table 2 Total uterine volume reduction percentage

\begin{tabular}{lll}
\hline Uterine volume reduction & Treatment group & \\
\cline { 2 - 3 } & Single fibroid & Multiple fibroids \\
\hline Pre embolization mean volume & $608.1 \mathrm{~cm}^{3}$ & $872.9 \mathrm{~cm}^{3}$ \\
Post embolization mean volume & $325 \mathrm{~cm}^{3}$ & $550 \mathrm{~cm}^{3}$ \\
Volume reduction & $46.5 \%$ & $37.4 \%$ \\
$P$ value & $0.01 *$ & \\
\hline
\end{tabular}

$\left.{ }^{*}\right)$ means statistically significant 
Table 3 Dominant fibroid volume reduction percentage

\begin{tabular}{lll}
\hline Fibroid volume reduction & Treatment group \\
\cline { 2 - 3 } & Single fibroid & Multiple fibroids \\
\hline Pre embolization mean volume & $270 \mathrm{~cm}^{3}$ & $247 \mathrm{~cm}^{3}$ \\
Post embolization mean volume & $138 \mathrm{~cm}^{3}$ & $136 \mathrm{~cm}^{3}$ \\
Volume reduction & $48.8 \%$ & $44.9 \%$ \\
$P$ value & 0.57 & \\
\hline
\end{tabular}

32.8 after UAE, and the HRQOL was 40.2 and 74, respectively (Table 4).

At 6 months follow-up at the MRI unit, complete infarction of the dominant leiomyoma was achieved in 18 patients $(85.7 \%)$ in group 1 , which had a statistically significant difference $\left(P\right.$ value $\left.0.03^{*}\right)$ compared with the group 2 in which complete infarction was achieved in 34 patients (57.6\%), as listed in Table 5.

Complications in group 2 included severe pain in two patients after discharge from hospital within 1 week that required readmission and IV analgesia, complete expulsion of a dominant fibroid in one patient, and infection in two patients, which was treated by IV antibiotics and hospital readmission.

Spasm of right uterine artery was experienced in one patient with a failed embolization from that side and successful embolization of the left uterine artery. At follow-up, successful fibroid infarction was achieved.

Complications in group 1 were expulsion of the fibroid in one patient, severe pain in one patient requiring readmission, and pain control.

\section{Discussion}

UAE has become a popular treatment option for women with symptomatic fibroids who desire uterine preservation and/or the avoidance of surgical intervention [7].

Our results have demonstrated that there was a significant reduction in the uterine volume between the studied groups with total volume reduction in $46.5 \%$ and $37.4 \%$ in group 1 and group 2 , respectively $(P=0.01)$. However, there was no significant difference in the reduction of the dominant fibroid volume between the two groups with volume reduction of $48.8 \%$ and $44.9 \%$ in group 1 and group 2, respectively (no significant difference, $P=0.57$ ).
Table 5 Dominant Fibroid infarction

\begin{tabular}{|c|c|c|c|c|}
\hline & \multicolumn{4}{|c|}{ Dominant Fibroid infarction } \\
\hline & \multicolumn{2}{|c|}{ Single fibroid group } & \multicolumn{2}{|c|}{ Multiple fibroids group } \\
\hline & $(n)$ & (\%) & $\overline{(n)}$ & (\%) \\
\hline $100 \%$ & 18 & 85.7 & 34 & 57.6 \\
\hline $99-90 \%$ & 2 & 9.5 & 16 & 27.1 \\
\hline $75-90 \%$ & 1 & 4.7 & 3 & 5 \\
\hline $50-74 \%$ & 0 & 0 & 2 & 3.3 \\
\hline$<50 \%$ & 0 & 0 & 4 & 6.6 \\
\hline$P$ value & $0.03^{*}$ & & & \\
\hline
\end{tabular}

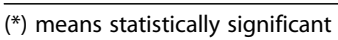

Das et al.'s [9] study revealed that the dominant fibroid volume mean reduction was $50 \%$ at 6 months, while the mean uterine volume reduction was $42 \%$ at 4 months.

- At 6 months MRI follow-up, complete infarction of the dominant leiomyoma was achieved in 18 patients $(85.7 \%)$ in group 1 , and complete infarction was achieved in 34 patients in group 2 (57.6\%). In the study of Richard et al. [13], complete dominant tumor infarction was maintained at 3 months in $82.1 \%$ of patients in the PVA UAE group.

- With the use of PVA as our main embolic agent, we achieved satisfactory clinical success. In group 1, the mean symptom severity score markedly decreased from 65.8 to 30.4 after treatment, and the mean HRQOL improved from 33.3 to 78.2 . While in group 2, the mean symptom severity score was 70.1 pre-procedure and 32.8 post procedure, and the HRQOL improved from 40.2 to 74 .

In a study by Das et al. in 2014 [9], HR-QOL improved from 39.1 pre UAE to 76.2 post UAE, and symptom severity score decreased from 55.9 to 26.6 before and after UAE, respectively.

All patients with uterine fibroids were treated by UAE using bilateral arterial femoral artery access. The mean screening time was $15.68 \mathrm{~min}$, the mean dose was 191 $\mathrm{mGy}$, and the dose area product (DAP) was = $2520 \mu \mathrm{Gym}^{2}$ with no significant differences detected with

Table 4 Mean symptom severity score and mean HR QOL

\begin{tabular}{llllll}
\hline & \multicolumn{2}{l}{ Single fibroid group } & & \multicolumn{2}{l}{ Multiple fibroids group } \\
\cline { 2 - 3 } & Mean symptoms severity score & Mean HR-QOL & & Mean symptoms severity score & Mean HR-QOL \\
\hline Pre UAE & 65.8 & 33.3 & 70.1 & 30.2 \\
Post UAE & 30.4 & 78.2 & 32.8 & 74 \\
Mean difference between Pre/Post & 35.4 & 44.9 & 37.3 & 33.8 \\
$P$ value & 0.67 & & & \\
\hline
\end{tabular}


respect to fluoroscopy time or radiation dose between the two groups.

In a study by Bratby et al. [14], elective bilateral arterial punctures were reported aiming to reduce patient irradiation by using pulsed fluoroscopy, avoidance of aortography, and simultaneous bilateral embolization.

The study results are limited by unequal number of patients in the study groups, so further study from multiple centers is recommended for better assessment.

\section{Conclusion}

UAE has high efficacy in reducing uterine volumes in the patients with a single fibroid. The results of UAE are improved in patients with single fibroids rather than multiple fibroids. UAE improved the quality of life whether patients had single or multiple fibroids.

\section{Abbreviations}

F: French; FSE: Fast spin echo; HR-QOL: Health-related quality of life questionnaire; IR: Interventional radiology; MRI: Magnetic resonance imaging; PVA: Polyvinyl alcohol; UAE: Uterine artery embolization

\section{Acknowledgements}

To all the participants for their cooperation and patience

\section{Authors' contributions}

AME suggested the research idea, ensured the original figures and data in the work, correlated the study concept and design, collected data in all stages of manuscript, and had the major role in analysis. ME and HE supervised the study with significant contribution to design the methodology, manuscript revision, and preparation. RM correlated the clinical data of patient and matched it with the findings, drafted, and revised the work. AK performed data analysis. All authors read and approved the final manuscript.

\section{Funding}

No funding sources

\section{Availability of data and materials}

The datasets used and analyzed during the current study are available from the corresponding author on reasonable request

\section{Ethics approval and consent to participate}

Informed written consents were taken from the patients. The study was approved by ethical committee of Tanta University Hospital, Faculty of Medicine number 30361/06/2015.

\section{Consent for publication}

Participants included in the research gave written consent to publish the data included in the study. Authors accepted to publish the paper.

\section{Competing interests}

The authors declare that they have no competing interests.

\section{Author details}

${ }_{1}^{1}$ Radio-diagnosis \& Medical Imaging Department, Faculty of Medicine, Tanta University, Tanta, Egypt. ${ }^{2}$ Diagnostic and Interventional Radiology Department, St George's University Hospital and NHS Foundation Trust, London, UK. ${ }^{3}$ Diagnostic and Interventional Radiology Department, Faculty of Medicine, Cairo University, Cairo, Egypt.
Received: 11 November 2019 Accepted: 23 April 2020

Published online: 15 May 2020

References

1. Zimmermann A, Bernuit D, Gerlinger C et al (2012) Prevalence, symptoms and management of uterine fibroids: an international internet-based survey of 21,746 women. BMC Womens Health 12:6

2. Donnez J, Dolmans M (2016) Uterine fibroid management: from the present to the future. Hum Reprod Update. 22(6):665-686

3. Deshmukh SP, Gonsalves CF, Guqlielmo FF et al (2012) Role of MR imaging of uterine leiomyomas before and after embolization. Radiographics. 32(6): E251-E281

4. Bulman JC, Ascher SM, Spies JB (2012) Current concepts in uterine fibroid embolization. Radiographics. 32(6):1735-1750

5. Dariushnia SR, Nikolic B, Stokes LS et al (2014) Quality improvement guidelines for uterine artery embolization for symptomatic leiomyomata. J Vasc Interv Radiol. 25(11):1737-1747

6. Manyonda IT, Bratby M, Horst JS et al (2012) Uterine artery embolization versus myomectomy: impact on quality of life-results of the FUME (fibroids of the uterus: myomectomy versus embolization) trial. Cardiovasc Interv Radiol. 35(3):530-536

7. Siddiqui N, Nikolaidis $\mathrm{P}$, Hammond $\mathrm{N}$ et al (2013) Uterine artery embolization: pre- and post-procedural evaluation using magnetic resonance imaging. Abdom Imaging. 38:1161-1177

8. Verma SK, Gonsalves CF, Baltarowich OH et al (2010) Spectrum of imaging findings on MRI and CT after uterine artery embolization. Abdom Imaging. 35(1):118-128

9. Das R, Rita Champaneria R, Daniels JP et al (2014) Comparison of embolic agents used in uterine artery embolisation: a systematic review and metaanalysis. Cardiovasc Intervent Radiol 37:1179-1190

10. Kroencke TJ, Kluner C, Hamm B, Gauruder-Burmester A (2007) Use of the 4F Rosch inferior mesenteric catheter in embolization procedures in the pelvis: a review of 300 cases. Cardiovasc Intervent Radiol. 30(2):268-272

11. Kroencke TJ, Scheurig C, Poellinger A et al (2010) Uterine artery embolization for leiomyomas: percentage of infarction predicts clinical outcome. Radiology 255:834-841

12. Das R, Gonsalves M, Vlahos I, Manyonda I, Belli AM (2013) MRI assessment of uterine artery patency and fibroid infarction rates 6 months after uterine artery embolization with nonspherical polyvinyl alcohol. Cardiovasc Intervent Radiol 36(5):1280-1287

13. Richard SG (2014) D., Mark R.A., Jeffrey M.I., et al. Comparison of polyvinyl alcohol microspheres and Tris-Acryl gelatin microspheres for uterine fibroid embolization: results of a single-center randomized study. J Vasc Interv Radiol 25:823-832

14. Bratby MJ, Ramachandran N, Sheppard N et al (2007) Prospective study of elective bilateral versus unilateral femoral. Cardiovasc Intervent Radiol. 30: 1139-1143

\section{Publisher's Note}

Springer Nature remains neutral with regard to jurisdictional claims in published maps and institutional affiliations.

\section{Submit your manuscript to a SpringerOpen ${ }^{\circ}$ journal and benefit from:}

- Convenient online submission

- Rigorous peer review

- Open access: articles freely available online

High visibility within the field

- Retaining the copyright to your article

Submit your next manuscript at $>$ springeropen.com 